\title{
Celiac plexus block in a patient with upper abdominal pain caused by diabetic gastroparesis
}

\author{
Young-Bok Lee and Won-Seop Kim \\ Department of Anesthesiology and Pain Medicine, Yonsei University Wonju College of Medicine, Wonju, Korea
}

Diabetic gastroparesis is characterized by delayed gastric emptying, which stems from long-standing diabetic neuromyopathy. The symptoms of gastroparesis include early satiety, nausea, vomiting, postprandial fullness and upper abdominal pain [1]. Here, we present the case of a patient with a 20 -year history of diabetes mellitus and a 2-year history of gastroparesis. The patient was suffering from frequent nausea, vomiting and upper abdominal pain, which remained unresponsive to medical treatments.

A 40-year-old woman was referred to our pain clinic by the department of nephrology with a chief complaint of intractable upper abdominal pain. She had been given a score of 9-10 on the visual analogue scale (VAS). The pain was described to be cramping and bitter as the patient remained in a knee-chest crouched position. She presented a known case of juvenile diabetes which had first been diagnosed at the age of 13. As the age of 38 , she had diagnosed with diabetic gastroparesis. She had been admitted for 3-4 days each month for upper abdominal pain management. At the time of the referral, her blood pressure was $120 / 80 \mathrm{mmHg}$ and her pulse rate was $100 / \mathrm{min}$. Her height and body weight were $151 \mathrm{~cm}$ and $38.4 \mathrm{~kg}$, respectively. The laboratory examinations revealed the following, $\mathrm{HbA1c}$, glucose, $\mathrm{BUN} /$ creatinine and albumin levels; 7.1\%, $131 \mathrm{mg} / \mathrm{dl}, 50 / 2.3 \mathrm{mg} / \mathrm{dl}$ and $4.8 \mathrm{~g} / \mathrm{dl}$, respectively. At the time of her final admission, the intractable pain overshadowed nausea and vomiting. As the pain characteristics showed a visceral origin, we decided to perform a thoracic epidural block in an attempt to block the sympathetic nerve in the upper abdominal area. The thoracic epidural block was performed using a paramedian approach at the T7-T8 level with $10 \mathrm{ml}$ of $1 \%$ mepivacaine. The patient fell asleep 5 minutes after the end of the nerve block procedure. After a 2-hour sleep, she was returned to the ward with no pain complaint. She was discharged pain-free the next day but returned to the hospital for recurrent abdominal pain 2 weeks later. On this admission, her VAS score was 6-7. She was referred to our department for a more sustainable form of pain control. This time, we decided to perform celiac plexus block with alcohol. A left celiac plexus block was performed with $10 \mathrm{ml}$ absolute alcohol first. The right-side celiac plexus nerve was suspended for the significant pain reduction. She was discharged from the hospital with no complications after 2 days. She experienced significantly reduced pain thereafter and was only been admitted to the hospital twice for recurrent nausea and vomiting over the 6-month follow-up period.

Gastroparesis is a functional disorder presenting delayed emptying of the gastric contents which is more prevalent in women. GI symptoms are present in $76 \%$ of diabetic patients, and $34 \%$ of patient reports abdominal pain [2]. The origin of this abdominal pain is a source of debate, but it is suggested that the symptoms are related to a neurogenic disturbance at the enteric nervous system level or the spinal/brain level [3]. Our patient had been suffering from nausea, vomiting and upper abdominal pain for 2 years. She had received strong pain medications including intravenous tramadol and meperidine on her admissions. To control the abdominal pain, opioids, tricyclic antidepressants, gabapentin and pregabalin had been suggested. Our patient was already suffering from chronic gastroparesis symptoms and was referred to our pain clinic for her intractable

Corresponding author: Young-Bok Lee, M.D., Ph.D., Department of Anesthesiology and Pain Medicine, Yonsei University Wonju College of Medicine, 20, Ilsan-ro, Wonju 220-701, Korea. Tel: 82-33-741-1536, Fax: 82-33-742-8198, E-mail: yblee@yonsei.ac.kr

(c) This is an open-access article distributed under the terms of the Creative Commons Attribution Non-Commercial License (http:// creativecommons.org/licenses/by-nc/3.0/), which permits unrestricted non-commercial use, distribution, and reproduction in any medium, provided the original work is properly cited. 
upper abdominal pain. This was our first experience with a patient suffering from intractable abdominal pain caused by diabetic gastroparesis. First, we decided to perform a thoracic epidural sympathetic nerve block to manage the pain. The thoracic epidural block has the advantage of blocking the nociceptive afferent fibers while maintaining the function of the craniosacral parasympathetic efferent fibers [4]. After remaining free from severe pain for 2 weeks, the patient returned to our hospital for recurrent abdominal pain. Following consultation with the nephrologist, a celiac plexus block using alcohol was performed for a more sustainable form of pain control. This procedure had a satisfactory pain control outcome and an acceptable level of pain was subsequently maintained. Nevertheless, it did not prevent or help manage the nausea and vomiting. The celiac plexus block has been shown to increase GI motility in addition to blocking the pain while in severe cases, hypermobility of the GI tract has led to diarrhea. We expected that the celiac plexus block would alleviate all of the symptoms of diabetic gastroparesis; however, the block only resulted in pain reduction. This could be a result of the chronicity of the symptoms in our patient. Parasympathetic neuropathy can also be contributing to these symptoms. Therefore, a splanchnic nerve block can also improve the upper abdominal pain [5]. In conclusion, we recommend further studies on the mechanism of abdominal pain in diabetic gastroparesis as it is imperative to maintain intensive pain management in these patients.

\section{References}

1. Shakil A, Church RJ, Rao SS. Gastrointestinal complications of diabetes. Am Fam Physician 2008; 77: 1697-702.

2. Koch KL. Diabetic gastropathy: gastric neuromuscular dysfunction in diabetes mellitus: a review of symptoms, pathophysiology, and treatment. Dig Dis Sci 1999; 44: 1061-75.

3. Hoogerwerf WA, Pasricha PJ, Kalloo AN. Schuster MM. Pain: the overlooked symptom in gastroparesis. Am J Gastroenterol 1999; 94: 1029-33.

4. Clemente A, Carli F. The physiological effects of thoracic epidural anesthesia and analgesia on the cardiovascular, respiratory and gastrointestinal systems. Minerva Anestesiol 2008; 74: 549-63.

5. Choi JW, Joo EY, Lee SH, Lee CJ, Kim TH, Sim WS. Radiofrequency thermocoagulation of the thoracic splanchnic nerve in functional abdominal pain syndrome. Korean J Anesthesiol 2011; 61: 79-82. 